\title{
CRehab: A Cloud-based Framework for the Management of Rehabilitation Processes
}

\author{
Habib M. Fardoun ${ }^{1}$, Abdulraham H. Altalhi ${ }^{1}$, \\ Antonio Paules Cipres ${ }^{2}$ \\ ${ }^{1}$ Information Systems Department \\ Jeddah, Saudi Arabia \\ King Abdulaziz University (KAU) \\ \{hfardoun, ahaltalhi\}@kau.edu.sa \\ ${ }^{2}$ University of Castilla-La Mancha (UCLM) \\ Albacete, Spain \\ apcipres@gmail.com
}

\author{
Jaime Ramírez Castillo ${ }^{1}$, Sergio Albiol-Pérez ${ }^{2}$ \\ ${ }^{1}$ itknowingness \\ Albacete, Spain \\ jaime.ram@gmail.com \\ ${ }^{2}$ Dpto. de Informática e Ingeniería de Sistemas \\ Universidad de Zaragoza \\ C. Ciudad Escolar S/N, 44003, Teruel, SPAIN
}

\begin{abstract}
- this paper presents a conceptual cloud-based framework with virtual and augmented reality support named "CRehab" for the management of rehabilitation processes. Studies show that rehabilitation wards, staff, patients, and families need systems that tackle administration, personal communication, and patient motivation processes effectively. To this end, CRehab employs cloud capabilities to address the first two concerns. Patient motivation is encouraged by the deployment of virtual rehabilitation environments with exercises that stimulate the patient. The main aim is to create feasible architecture for use in multiple types of rehabilitation, regardless of diagnosis, environment, patient, or any other factors.
\end{abstract}

Keywords: Cloud Computing, Rehabilitation Frameworks, Rehablitation Processes, Virtual Reality.

\section{INTRODUCTION}

Clinical rehabilitation is carried out on a daily basis by hard-working staff and patients. This research focuses on Cloud Computing, and Augmented Reality, and how it can improve the process of management, recovery, and the collaborative relationship between clinic staff and patients. The continuous development of new technology has produced some amazing rehabilitation facilities; however, more research needs to be done on patient-therapist collaboration, staff communication, and subjective factors such as patient motivation.

This article introduces the design of a cloud-based framework to enhance and ease the rehabilitation process. This is done by moving computing resources (patient data, reports, assessments, motorizations, etc.) to the Cloud and by the provision of augmented reality that gives users suitable interfaces to meet the requirements of each patient. The research includes details of how the framework improves the collaboration between clinic staff and patients by using the systems to encourage patients' motivation. Cloud services create a distributive system that makes interaction between all users easier, this includes therapists, specialists, patients, and families. The main aim of the project is to design and develop the reference framework for rehabilitation, which could then be adapted for use in any rehabilitation environment.

We have organised the article in sections. Section II outlines rehabilitation processes enriched by using cloud services and augmented reality. In Section III we explain the objectives of our framework and describe the architecture. Section IV is a brief case study and Section V details the conclusions and proposals for future work.

\section{REHABILITATION MEETS ClOUd COMPUTING}

\section{A. Rehabilitation}

This paper focuses on the creation of a framework to support and improve the rehabilitation process. Factors for successful patient recovery include patient motivation [1] [2], which is important and dependent on stimulation and the provision of appropriate information. The working alliance relationship between the patient and the healthcare professional, patients identified as highly motivated for rehabilitation have been found to align themselves more closely to the aims and methods of rehabilitation professionals, and are more likely to understand the nature and purpose of their rehabilitation, when compared to those with low motivation [3]. Active participation in the goal identification process, fluent partnership with the therapist and the provision of information about the rehabilitation process has been reported as positive motivational and empowering factors [4] [5]. Therefore, it is important to involve the patient in a communicative and informative environment.

Studies of the rehabilitation process show that there is room for improvement with regard to collaboration and communication among patients and clinic staff. Healthcare professionals and social workers are rarely asked for their opinions on patient condition, even though they spend more time with patients than other team members. It is important that these professionals voice their opinions in order to develop a more effective rehabilitation process [6] [7]. Healthcare professionals play a key role in patient care and so should be involved in the communication flow [8] [9]. 
A rehabilitation programme comprises a series of steps, describing the treatment to be applied and the variables to be adjusted to fit the patient profile. A consultant or specialist is in charge of the programme, makes decisions, and recognises the consequences. For small injuries, patients are given exercises that they can do at home, as preventative mechanisms or recovery routines. A typical programme includes:

- Initial consultation for physical and psychological evaluation.

- Development of a customised therapeutic process.

- Follow-up appointments (habit control and assessment of difficulties).

- Weekly sessions of physical activity, supervised by the Sports Medicine Service to improve body image, selfesteem, strength, and stamina.

- Final session: report delivery, conclusions, and recommendations.

\section{B. Cloud Computing}

Cloud computing is a recent phenomenon, linked to Web 2.0. The term refers to a collection of different services and resources stored on secure servers, available remotely at any time, from anywhere and accessible instantly. The user has access to their files and programs stored in a, delocalized and virtual environment (hence the name Cloud). Resources are permanently at the disposal of the user, regardless of time and location [10]. Documents are not physically held on one's own computer but are retrieved via the internet. Working in the cloud means that users do not have to rely on a program, an operating system, or even a particular location any more. To start working in the Cloud, all that is required is a device with an internet connection.

\section{Cloud computing applied to rehabilitation}

The great advantage of the "Cloud Rehabilitation" is that information can he shared in real time and multiple users can access and share the same file [11]. For example, a rehabilitation session could be shared between consultants, therapists, and patients at the same time [12]. Consultants could simultaneously inspect patient condition reports, view and edit rehabilitation exercises and organise the shared information to get a broader view of patient progress, using data from different sources [13]. Patients could access the data necessary for their exercises, from home. All this is based in a collaborative and communicative environment. Using Web 2.0 medical applications, the rehabilitation cloud could hold all patients' medical records and information about the rehabilitation process and be accessible to all involved, namely patients, families, therapists, healthcare professionals, social workers, specialists and consultants and could support and enhance rehabilitation [14] [15].

\section{CREHAB FRAMEWORK}

\section{A. CREHAB Definition}

As seen in the previous section, there are several studies and many ideas already developed to support patient rehabilitation by use of customised interaction devices and virtual or augmented reality. In general, research has focused on how to improve patient performance in therapy sessions by enabling improved interaction, updating robots performance, or designing better virtual environments. However, action on important subjective factors has been overlooked. Issues that need to be addressed are improved communication between those involved in the process, the transparency, and availability of information, and patients' motivation. Assessment and patient mobility is one area that could be improved, for example, when the patient interacts with devices, the therapist is not able to monitor patient's actions at the time. It is important to remember that one member of clinic staff can be in charge of many patients, each one with different records, making keeping track of all of them quite difficult. Having a proper system to monitor of all of them can help.

Projects previously reviewed targeted specific problems, whereas our proposal provides a generic solution in the form of a cloud-based framework application. Given the heterogeneity of rehabilitation projects, communication gaps among clinic staff and patients, and the possibilities offered by virtual and augmented reality, the proposal focuses on these three main areas. Firstly, it aims to consolidate and standardise all the rehabilitation methods, processes, and scopes. Secondly, Cloud services provide clients and professionals with robust tools to enhance communication, build trustworthy relationships, and manage relevant data in an accurate and timely way. Finally, augmented and virtual reality modules/plugins can be deployed easily to patient-customised environments. The system will be adaptable enough to manage any kind of rehabilitation therapy, by defining an information-based approach, which is patientcentred and human-aware, by helping any member of staff to voice their opinions and share information, regardless of their professional level. The objective is to gather and offer as much information as possible about the patient rehabilitation process, as the more information is readily available, the easier and more efficient it is for staff to assist the patient.

Clinic staff will be able to prepare rehabilitation exercises in advance. The system would include high-level customisation features, broad enough to manage any rehabilitation programme. Systems like those reviewed in the previous section and forthcoming projects could use the platform. The framework would enable easy access to real-time data as well as data on the patients' previous history, and is flexible enough for use as a management platform for rehabilitation projects. In order to do all of this, the following is required:

- Provision of statistical data to both therapist and patient, in order to assess progress of the exercise programme.

- The facility to customise the system for each patient profile and to save the settings. A fast and useable interface to facilitate everyday tasks.

- Cloud based architecture to allow patients to carry out their exercises from anywhere.

- Diagnosis and recovery processes to be carried out without requiring the physical presence of the user, to avoid moving patients to particular wards, allowing them to stay at home, thus improving quality of life. 
- Sensorial recovery in order to extend this solution to other kinds of recovery, such as physical activities for disabled students in schools [16] or functional recovery for the elderly [17] as a keep-fit process, rather than intensive rehabilitation.

- A plugin installation module, featuring customized virtual environments for rehabilitation.

- In order to perform functional testing, a videogame that implements physical rehabilitation will be prototyped. Depending on the kind and degree of disability, the videogame would be responsive and adaptable to suit the patient. This makes it suitable for a wide range of patients. The two main aims of this are to create a stimulating environment to motivate patients and to assist therapists to treat and monitor their patients in a customised way.

The solution is not restricted to rehabilitation centres and includes family homes, schools, or rest homes. In addition, it should be possible to extend its use beyond the scope outlined and for it to become a generic tool targeted at traditional recovery methods. Activities of Daily Living (ADLs) would also be enhanced.

\section{B. CREHAB Architecture}

The system architecture, covering all requirements and services is based on the Cloud, as illustrated in Figure 1.

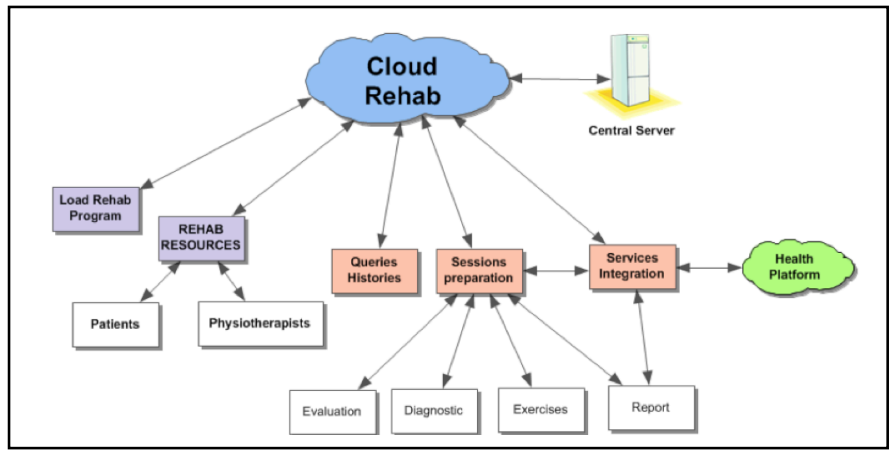

Figure 1. CREHAB Framework architecture

Figure 1 depicts the services related to the rehabilitation process: purple entities represent services that are accessible to patients; in red, we have platform specific services for specialists; green represents health platforms such as public administration health systems. The system is comprised of the following modules:

- Load Rehab: After session descriptions, exercises and guidelines for the patient are created; the system proceeds to load them to the framework. The loading process can be performed either online or by retrieving the data from a physical drive, such as a Universal Serial Bus (USB) drive. From here on, every patient action that takes place in the system can be monitored.

- Rehab Resources: The platform features $\left(\mathrm{CRUD}^{1}\right)$ with plans of patient exercises for users to access easily,

\footnotetext{
${ }^{1}$ Create, Read, Update and Delete
}

tools to construct keep-fit timetables that promote healthy living, and a search facility. These services are available publicly to both patients and clinic staff. Because of this, settings for each case will be different (class specialisation). Patient interfaces focus on how to do the exercises, whereas therapists access an administration site where they will be able to browse patient profiles, exercises, and other relevant information. Parameter settings will be stored in the system (in the Cloud), because of the configuration of each centre, how methods are defined, and how userinteraction statistics are used.

- Query Histories. Shows patient profiles. Clinic staff are granted access to patient profiles in order to look for previous rehabilitation episodes. It also provides services in an administration panel, enabling patient management, registrations and removal, and any other requirement the application might have.

- Rehab Session Set-up: This is the working environment of therapists, where they prepare sessions, exercises, and develop the diagnosis and evaluation process; generate final reports, schedules, and notifications.

- Services Integration: this publishes framework functions as web services for integration with other servers. The functions are patient data reception, report publication, management and integration configuration tasks.

The architecture outlines the requirements that a system must comply with to allow the game exercises to be loaded on the users' device. A deployment structure is required to connect commercial devices to the games, because rehabilitation games do not exist elsewhere as a type of game. The objective is to provide the specialists with a set of games to deliver to the user customised or to combine them to fit the patient profile.

The system to create exercises for users would be online, in a developed environment. Each game would be focused on one or more problems and a "wizard" would assist the user to create the game. This platform is intended to treat clinical patients, who do the exercises from home or from another suitable place. Additionally, we could offer a personal assistant service to non-clinical patients, as a way to keep fit.

\section{CASE OF STUDY}

In the case study, the therapist carries out a rehabilitation process for an impaired child in a school. The centre has all the necessary qualified personnel for the development and motorization of the exercises. Figure two shows the screen where the therapist can create tables with exercises for each patient. In this case, the therapist is creating a simple manual for daily use by a student with a motor disability. The interface is based on a validated working experience [18].

The interface allows the specialist to carry out the necessary adjustments for each patient's sessions. The following options are available:

- Pre-inscription: the screen where exercise sessions are created. 
- Report: this allows the generation of progress reports. Reports are digitally signed to ensure privacy and fully compliant with other medical Enterprise Resource Planning (ERP).

- Diagnostic: this section features the management of the complete medical diagnosis and profiles for each patient.

- Monitorization: here, clinic staff can consult patient recovery records, either historical or real-time.

- Resources: a complete list of resources available to staff.

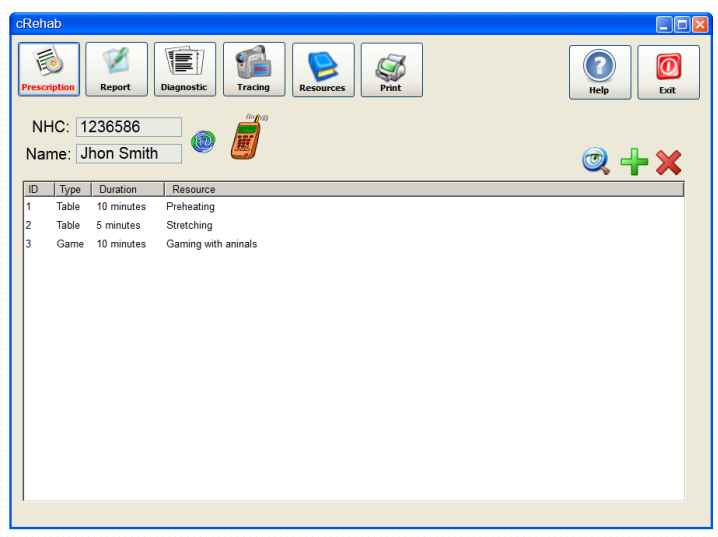

Figure 2. Exercise Schedule User Interface

\section{CONCLUSIONS AND FUTURE WORK}

In this paper, we have introduced a system for medical rehabilitation using Cloud technology and virtual and augmented reality. The system is a Cloud-based customisable platform for the management of rehabilitation data and the delivery of virtual environments for recovery exercises. It has been designed with structural similarities to educational environments to strengthen information analysis and to support evaluation and motorization in rehabilitation programmes.

The large variety of commercial devices available, make it necessary to create a platform that can effectively handle rehabilitation games and generated data. It is flexible enough to allow for the easy creation, installation, and management, of the deployment of the games to every type of device. An integration layer should be developed to act as Application Programming Interface (API) between devices and the framework.

Future work will focus on the development of the system prototype. This includes creating the Cloud structure featuring the modules described and developing a simple virtual augmented application to test how the system installs and deploys it to patient devices. We hope our experience and knowledge of Cloud-systems and user interfaces will help to develop tools that improve rehabilitation models.

\section{REFERENCES}

[1] M. Schönberger, F. Humle, T.W., "Teasdale Subjective outcome of brain injury rehabilitation in relation to the therapeutic working alliance, client compliance and awareness," Brain Inj, 20(12), pp. 1271-82, 2006

[2] ] W. E. Peschken, and M. E. Johnson, "Therapist and client trust in the therapeutic relationship," Psychotherapy Research, 7(4), pp. 439-447, 1997.

[3] Atwal, A. and Caldwell, K. (2005). Do all health and social care professionals interact equally: a study of interactions in multidisciplinary teams in the United Kingdom. Scandinavian Journal of Caring Sciences, 19: 268-273. doi: 10.1111/j.1471-6712.2005.00338.x.

[4] Fardoun, H. M., Antonio Paules Ciprés, Daniyal M. Alghazzawi. CSchool - DUI for Educational System using Clouds. Proceedings of the 2nd Workshop on Distributed User Interfaces: Collaboration and Usability. In conjunction with CHI 2012 Conference Austin, Texas, USA. ISBN-10: 84-695-3318-5. pp: 35-39. AUSTIN, TEXAS, USA. May 052012.

[5] Gens, F., 2008. "Defining "Cloud Services" and "Cloud Computing". http://blogs.idc.com/ie/?p=190. (Accessed22.8.2010).

[6] A.M. Hall, P.H. Ferreira, C.G. Maher, J. Latimer, M.L.Ferreira, "The influence of the therapist-patient relationship on treatment outcome in physical rehabilitation: a systematic review," Phys Ther,90(8), pp.1099$110,2010$.

[7] ] T. Munder, F. Wilmers, R. Leonhart, HW Linster, J. Barth, "Working Alliance Inventory-Short Revised (WAI-SR): psychometric properties in outpatients and inpatients," Clin Psychol Psychother, 17(3), pp. 231-9, 2010.

[8] Habib M. Fardoun, Antonio Paules Cipres, Daniyal Alghazzawi (2012). "TabletNet: Utility, Usability and User Interface Quality (P)", 13th International Congress on Human Computer Intercation (Interacción 2012), Octobor 3-5, 2012, Elche, Spain, AIPO.

[9] Jones, M., O'Neill, P., Waterman, H. and Webb, C. (1997). Building a relationship: communications and relationships between staff and stroke patients on a rehabilitation ward. Journal of Advanced Nursing, 26: 101110. doi: 10.1046/j.1365-2648.1997.1997026101.x.

[10] G.C. Burdea, "Rubber ball to cloud rehabilitation musing on the future of therapy," Virtual Rehabilitation International Conference, pp.50,50, 2009

[11] W. Mingyu, Z. Qiang, Z. Weimo, Y. Jijiang, W. Qing, Q. Weiyi et al. "Remote rehabilitation model based on BAN and cloud computing technology," e-Health Networking, Applications and Services (Healthcom), pp.119,123, 2012

[12] Maclean N, Pound P, Wolfe C, Rudd A. A qualitative analysis of stroke patients' motivation for rehabilitation. BMJ. 2000;321:1051.

[13] R.E.E. Anderson, S.H. Spence, C.L. Donovan, S. March, S. Prosser, J. Kenardy, "Working alliance in online cognitive behavior therapy for anxiety disorders in youth: comparison with clinic delivery and its role in predicting outcome," J Med Internet Res, 14(3), e88, 2012.

[14] L.B. Nordgren, P. Carlbring, E. Linna, G. Andersson, "Role of the Working Alliance on Treatment Outcome in Tailored Internet-Based Cognitive Behavioural Therapy for Anxiety Disorders: Randomized Controlled Pilot Trial," JMIR Res Protoc, 2(1), e4, 2013

[15] Maclean N, Pound P, Wolfe C, Rudd A,. (2000) The Concept of Patient Motivation. A Qualitative Analysis of Stroke Professionals' Attitudes.

[16] Seelman K. Transition from a Medical Model to an Integrative Model. University of Pittsburgh. 2003

[17] Wressle E, Eeg-Olofsson A, Marcusson J,, Henriksson C,. Rehabil J Med. 2002 Jan ;34 (1):5-11. Improved client participation in the rehabilitation process using a client-centred goal formulation structure.

[18] Consejo Europeo de Medicina Física y Rehabilitación. Documento de la Unión Europea de Médicos Especialistas (UEMS). Currículum de estudios y conocimientos teóricos para la obtención del Diploma del Consejo Europeo de Medicina Física y Rehabilitación (versión 2005). 